\title{
Der Allgemeine deutsche Sprachverein und seine Zweigvereine in Mähren ${ }^{1}$
}

\author{
Libuše SPÁČILOVÁ
}

\begin{abstract}
The General German Language Society (Der Allgemeine deutsche Sprachverein) and its affiliated societies

In the first part, the study presents a brief characteristics of the development of the Modern High German language after 1650 from the point of view how other languages influenced its vocabulary and analyses numerous loan words. The second part deals with the origins and the first activities of the General German Language Society (from 1885 on) which became the leader in the field of the institutionalised ideological fight against foreign words in the central German language area. The third part introduces the research results of the affiliate societies which were founded in Moravia, one of the part of the Lands of the Bohemian Crown. Both the information in the journal of the General German Language Society and archive documents in three Moravian archives (in Brno, Jihlava and Nový Jičín - the only Moravian cities where affiliate societies were founded) show that their existence was rather a peripheral issue in these cities. As opposed to this, affiliated societies were very active in the cities of northern Bohemia. At the end of the study, the author considers the reasons which caused this kind of situation in Moravia.
\end{abstract}

Keywords: General German Language Society, foreign word, affiliated societies, Moravia, Modern High German language

DOI: doi.org/10.15452/StudiaGermanistica.2021.28.0006

\section{Einleitung}

Die Tätigkeit des Allgemeinen deutschen Sprachvereins (im Weiteren ADSV) ist heutzutage nur selten Objekt linguistischer Untersuchungen; daher haben auch manche Germanisten und Studierende Germanistik in Tschechien nur eine ungenaue Vorstellung von den Aktivitäten dieses Vereins. Insbesondere ist nicht bekannt, dass es auch außerhalb des Deutschen Reichs Zweigvereine des ADSV gab, so auch in den böhmischen Ländern. Diese Studie setzt sich zum Ziel, die Aktivitäten des ADSV kurz zu skizzieren und das Wirken der Zweigvereine auf dem Territorium Mährens,

\footnotetext{
Diese Studie entstand im Rahmen des Forschungsprojekts IGA_2020_029 „Der deutsche Sprachpurismus und dessen Widerspiegelung in der Lexikographie und deutschsprachigen Periodika in Böhmen und Mähren“, das in den Jahren 2020-2021 an der Philosophischen Fakultät der Palacký-Universität Olomouc realisiert wird.
} 
einem Bestandteil der böhmischen Länder, vorzustellen. Zugleich will sie die Fragen beantworten, wie intensiv die Aktivität dieser Zweigvereine in Mähren war.

\section{Die neuhochdeutsche Sprache nach dem Jahre 1650}

In Deutschland entwickelte sich vom späteren 16. Jahrhundert bis zur Mitte des 17. Jahrhunderts eine partielle Vielsprachigkeit; neben Latein, Italienisch und Spanisch war vor allem Französisch eine häufig gebrauchte Sprache. Diese damals modische Vielsprachigkeit wurde auch durch viele literarische Übersetzungen unterstützt, in denen fremdsprachliche Ausdrücke oft beibehalten blieben und die so einen bedeutenden Beitrag zur Sprachmischung leisteten. Zu den Hauptgründen dieser sprachlichen Übernahmen gehörten mehrere Faktoren: die überseeischen Entdeckungen, die damit zusammenhängenden Kolonisierungen und die damit zusammenhängende adressatenspezifische Sprachenwahl im Geschäftsverkehr - beispielsweise kommunizierten süddeutsche Kaufleute mit Venetianern und Genuesern auf Italienisch (vgl. Polenz 1994:59). Diese sprachlichen Veränderungen wurden als eine Beeinträchtigung des Zustands und des Gebrauchs der deutschen Sprache wahrgenommen. Die Ursachen dafür, warum die deutsche Sprache damals unterdrückt und verstümmelt wurde, fasste der deutsche Dichter und Begründer des Pegnesischen Blumenordens Georg Philipp Harsdörffer (1607-1658) um die Mitte des 17. Jahrhunderts in der Schrift Der Teutsche Secretarius in sechs Punkten zusammen (Harsdörffer 1656:142-143):

„1. [...] der Gottesdienst [ist] vor Jahren im Latein verrichtet worden [...].

2. Die zweite Ursache schreibe ich den Gelehrten zu / welche was besonders haben / und ihnen mit ihrem eingeschalteten Latein / ein hohes Ansehen machen wollen: sogar / dass die Gewohnheit bei Gerichten einführt [...].

3. Hierzu ist das leidige Kriegswesen geschlagen / welches uns eines teils Italienische / anders teils Französische Wörter aufgetrungen [...].

4. Ferners haben zu dieser Sprachverderbung nit wenig geholfen die Kauf-und Handelsleute, welche fremde Waren / fremde Wörter / das italienische Buchhalten [...] eingeführet / und eine besondere Art zuschreiben angenommen $[\ldots]$.

5. Die fünfte Ursach ist denen beizumessen / welche die deutsche Sprache zu erheben und auszuüben vermeinen / der Sache aber zu wenig oder zu viel gethan [...].

6. Sechstens könnte auch für eine wichtige Ursache angeführet werden / daß sie neulich erst / von dreissig / vierzig Jahren hero / etlich wenige befliessen / der Grund sinkenden deutschen Sprache mit gesamt Handbietung unter die Arme zu greifen [...].“

Im 17. Jahrhundert beginnt zugleich die Geschichte der deutschen Sprachreinigung und Sprachpflege, die damals eng mit der Gründung und Tätigkeit der Sprachgesellschaften verbunden ist. Mitglied der ersten und bedeutendsten dieser Gesellschaften, der Fruchtbringenden Gesellschaft, später in Palmenorden umbenannt, war auch Georg Philipp Harsdörffer, ${ }^{2}$ der im Jahre 1644 in seiner ,Schutzschrift für die Teutsche Spracharbeit' die Ziele der Spracharbeit vorstellte, die sich in folgenden Schlüsselwörtern widerspiegelten: Sprachreinheit, Sprachschönheit, Sprachrichtigkeit, vollständige Wörterbücher als Sammlungen aller Stammwörter, Fachwörterbücher als Sammlungen von Fachwörtern und Übersetzungen ohne Fremdwörter (vgl. Eggers 1986:245). Neben dem Ausdruck Sprachreinigung wurde in der deutschen Sprachgeschichte auch der Terminus Sprachpurismus verwendet. $^{3}$

Vgl. ausführlicher in der Monographie von Markus Hundt (2000).

Dieser Begriff kommt dem modernen Begriff ,Sprachkritik` nahe; gemeint ist der allgemeine Sprachpurismus seit der Humanistenzeit und eine sprachnationalistische Triebkraft bei der Entstehung von Nationalbewusstsein und Nationalstaat in Deutschland. Der in diesem Sinne benutzte Sprachpurismus muss begrifflich und historisch vom Begriff „Fremdwortpurismus“ unterschieden werden, der ,für den seit der Napoleonzeit frühnationalistisch belebten, nach der Reichsgründung 1871 vereinspolitisch institutionalisierten ideologischen Kampf gegen sog. ,Fremdwörter“ reserviert wird“ (vgl. Polenz 1999:264-265). 
Gegen Ende des 17. Jahrhunderts ist vor allem der französische Einfluss angewachsen. Die Verwendung der Gallizismen im Deutschen kann man in der Alamodezeit unter und nach Ludwig XIV. finden; in den Jahren 1650-1770 orientierten sich Repräsentanten des Adels und des höheren Bürgertums nach der Mode (fr. á la mode) von Paris und dem französischen Hof. Entlehnte Ausdrücke erschienen in vielen Gebieten des Alltagslebens - im Bereich Kleidung und Haartracht (Mode, Kostüm, Weste, Parfüm, frisieren, Perücke), Küche (Bouillon, Omelette, Ragout, Torte, Serviette, Tasse, delikat), Wohnkultur (Balkon, Salon, Hotel, Gardine, Sofa, Büffet) und Gesellschaftsleben (amüsieren, Maskerade, Billard, Karusell, Promenade), aber auch bei den Anredeformen (Madam, Mademoiselle) und Verwandtschaftswörtern (Papa, Mama, Onkel, Tante, Cousin, Cousine). Die Gefahr für das Deutsche bestand nicht mehr ,nur in der ,Sprachmengerei“, in dem Eindringen einer, wenn auch großen, so doch immerhin beschränkten Fremdwörtermenge in den deutschen Wortschatz, sondern in der völligen Verdrängung des Deutschen aus dem gesellschaftlichen Verkehr der Gebildeten“ (Polenz 2009:101). Französische Kulturschemata blieben bis zur wilhelminischen Zeit weiterhin wichtig, worin einer der Gründe bestand, warum die Sprachreinigung im ausgehenden 19. Jahrhundert und Anfang des 20. Jahrhunderts so aktuell war.

In der deutschen Sprachgeschichte entfaltete sich der Sprachpurismus in drei Phasen. Für die erste Phase, die vom 17. bis zum 18. Jahrhundert verlief und in der die Spracharbeit unterstützt wurde, war es wichtig, eine selbstständige deutsche Literatur, die gegen die Vorherrschaft des Lateinischen und Französischen wirken konnte, zu entwickeln (Kirkness 1998:408). In der sog. Übergangsphase von 1789 bis 1819 wurde der Purismus, der weiterhin kein großes Echo in der Öffentlichkeit fand, viel in akademisch gebildeten Kreisen, vor allem in Berlin und Braunschweig, diskutiert (Kirkness 1998:410). Hauptrepräsentant der Sprachreinheitsbestrebungen war damals Joachim Heinrich Campe (1746-1818), ${ }^{4}$ der - durchaus aufklärerisch gestimmt - sich intensiv der Verdeutschung von Fremdwörtern widmete und mehr als 3000 neue Wörter entstehen ließ. Sein Verdeutschungswörterbuch (Campe 1801, ${ }^{2}$ 1813) steht am Anfang einer späteren Tradition von Sonderwörterbüchern zur Erklärung und Verdeutschung der Fremdwörter, die im ausgehenden 19. Jahrhundert kulminierte. ${ }^{5}$ Nach der Konstituierung einer nationalistischen Richtung, deren Vertreter z. B. der „Turnvater“ Friedrich Ludwig Jahn (1778-1852) war, diente der Purismus zu einem (kultur-)politischen Zweck - die Fremdwörter wurden als Symptome des verderblichen fremden Einflusses auf Deutschland wahrgenommen. Seit dem 19. Jahrhundert entwickelte sich der Purismus in Deutschland immer mehr unter nationalistischen Vorzeichen (Kirkness 1998:412). Während die Bewegung in der zweiten Etappe zu Beginn des 19. Jh. eher Sache einzelner Interessierter war, kam es in der dritten Etappe, die ihren Anfang in den 70er Jahren des 19. Jahrhunderts hatte, im Deutschen Reich zur Institutionalisierung des Purismus, was u. a. die Reinigung der Amtssprache des Bauwesens, der Eisenbahn, der Postsprache etc. mit sich brachte. Die Fremdwortjagd wurde seit der Mitte der 80er Jahre des 19. Jh. zu einer verbreiteten Volksbewegung (Kirkness 1998:413). ${ }^{6}$

Campe wirkte als Verleger in Braunschweig, wo später auch Herman Riegel tätig war.

$\mathrm{Zu}$ Campes durchgesetzten Verdeutschungsvorschlägen gehörten z. B. altertümlich (anstelle von antik), Erdgeschoß (anstelle von Parterre), Ergebnis (anstelle von Resultat) oder Feingefühl (anstelle von Delikatesse). Unter seinen Vorschlägen, die nicht erfolgreich waren, kommen beispielsweise Weiberhof (anstelle von Harem), Zwangsgläubiger (anstelle von Katholik), Scheidekunst (anstelle von Chemie), Gesundheitsprobe (anstelle von Quarantäne) vor (vgl. Polenz 1994:131-132).

$6 \quad$ Im letzten Drittel des 19. Jahrhunderts verstärkten puristische Bestrebungen nicht nur in Deutschland, sondern auch in den böhmischen Ländern, denn Sprachpurismus gab es auch in der tschechischen Schriftsprache. In einer Periodisierung, die von Miloš Weingart im Jahre 1934 vorgeschlagen wurde, besteht der tschechische Sprachpurismus aus neun Etappen; Milan Jelínek präzisiert diese Gliederung und findet insgesamt zwölf Phasen (vgl. Jelínek 2007:541-542). Gerhard Engelhardt befasste sich in seiner Studie ,Český a německý purismus z konce 19. století “ [Der tschechische und der deutsche Purismus vom Ende des 19. Jahrhunderts] mit der Frage, ob es sich um einen reinen Zufall oder um eine Folge der Kontakte zwischen den tschechischen und deutschen Puristen handelte (vgl. Engelhardt 2001:235). 


\section{Die Gründung des Allgemeinen deutschen Sprachvereins}

Der Sprachpurismus war eng mit der Tätigkeit der Sprachgesellschaften (17. Jh.) und der Deutschen Gesellschaften (18. Jh.) verbunden. Ein wichtiges Ereignis in der zweiten Hälfte des 19. Jahrhunderts auf diesem Gebiet stellte die Gründung des Allgemeinen deutschen Sprachvereins dar. Die Initiative dazu übernahm in den 80er Jahren der Braunschweiger Kunsthistoriker und Museumsdirektor Herman Riegel (1834-1900), der im Jahre 1883 die Schrift ,Ein Hauptstück von unserer Muttersprache' mit dem Untertitel ,Mahnruf an alle national gesinnten Deutschen“ verfasste und sich dadurch als Wortführer der Fremdwortbekämpfung präsentierte. In seiner Schrift charakterisierte er den damaligen Zustand der deutschen Sprache und dabei führte er mehrere Beispiele aus der Belletristik an, um der Öffentlichkeit die starke Beeinflussung der deutschen Sprache durch das Französische zu zeigen; beispielsweise verwies er auf die deutschen Bauerngeschichten ,Erzählungen aus dem Ries ${ }^{\circ}$ von Melchior Meyr (1810-1871) und zählte viele in diesen Texten vorkommende Entlehnungen auf wie respectabel, existiren, ignoriren, Refrain, Galanterie, alteriren, Plaisir, Repräsentant, Contrast, commod, geniren, expliciren, Scrupel usw. (Riegel 1883:7).

Riegels puristische Bestrebungen waren zum Teil in seiner regionalen Herkunft begründet - er stammte aus dem nahe an der französischen Grenze gelegenen Breisgau, und die Frankreichfeindschaft motivierte manche seiner Äußerungen:

„Ich eifere nicht blind gegen die Fremdwörter überhaupt, wohl aber mit Nachdruck gegen das Übermaß und die Geschmacklosigkeit in der Anwendung derselben, namentlich der französischen. Mein Grundsatz ist: Kein Fremdwort für das, was deutsch ausgedrückt werden kann“(Riegel 1883:4-5).

In seiner Programmschrift charakterisiert er kurz und bündig sein Vorhaben - er möchte „keine blinde Reinigungswut, sondern eine vernünftige, überlegte und allmähliche Besserung“ der deutschen Sprache initiieren (Riegel 1883:6). Mit seinem Rundschreiben im August 1885 wandte er sich an bedeutende Repräsentanten des öffentlichen Lebens, und mit zwanzig wichtigen Persönlichkeiten versandte er im August 1885 einen Aufruf zur Gründung eines Sprachvereins. Er regte damit die Entstehung des ADSV an, der an frühere puristische Leistungen der zweiten Hälfte des 19. Jahrhunderts anknüpfen sollte.

Beispielsweise führte der Reichspostmeister Heinrich von Stephan im Jahre 1874/75 für das neu gegründete Institut der Reichspost insgesamt 671 neue Posttermini ein und ersetzte dadurch französische Ausdrücke wie postlagernd $\leftarrow$ poste restante oder Einschreiben $\leftarrow$ recomandé (Blume 2013:10); als ein anderes Beispiel für eine Sprachreinigung kann man den Germanisten und Gymnasiallehrer Hermann Dunger (1843-1912) anführen, der 1882 das ,Wörterbuch von Verdeutschungen entbehrlicher Fremdwörter der von dem Großen Generalstabe, im Postwesen und in der Reichsgesetzgebung angenommenen Verdeutschungen' herausgab (z. B. Weltbürger $\leftarrow$ Kosmopolit; Bittsteller, Bewerber $\leftarrow$ Petent; Wörterbuch, Wortsammlung $\leftarrow$ Vokabular; Dunger 1882, passim). Die Autoren konkreter Vorschläge für Verdeutschungen gingen pragmatisch vor und berührten fast alle wichtigen Gebiete des öffentlichen Lebens - u. a. das militärische Fachvokabular, das Rechtswesen und das Eisenbahnwesen.

Ein bedeutendes Ereignis stellte die Gründung des ersten Zweigvereins des ADSV dar. Hermann Dunger initiierte am 10. September 1885 in Dresden die Entstehung einer solchen Organisation. Dieser Tag gilt als das offizielle Gründungsdatum des ADSV. Nach zwei Jahren seiner Existenz, im Jahre 1887, hatte der Sprachverein 6500 Mitglieder und 91 Zweigvereine im ganzen Reich. Der neu gegründete ADSV lässt sich „bei rein systematischer Bestimmung“ [...] ähnlich wie z. B. die Fruchtbringende Gesellschaft des Barock als Sprachgesellschaft bezeichnen (Gardt 1998:332). ${ }^{7}$ Herbert Blume zufolge war der Sprachverein ,ein Kind des Nationalismus der wilhelminischen Epoche“ (Blume 2013:3). Eine wichtige Tat war die Herausgabe der ,Zeitschrift des Allgemeinen

Andreas Gardt (1998:332) geht von der Voraussetzung aus, Sprachgesellschaften sind „diejenigen Sozietäten, bei denen die Sprache im Fokus aller Aktivitäten steht“. 
deutschen Sprachvereins' ab dem Jahre 1886. ${ }^{8}$ Die erste Nummer der Zeitschrift veröffentlichte auf der ersten Seite die Hauptziele des Vereins:

„Der allgemeine deutsche Sprachverein ist ins Leben getreten, um

1. die Reinigung der deutschen Sprache von unnöthigen fremden Bestandtheilen zu fördern,

2. die Erhaltung und Wiederherstellung des echten Geistes und eigentümlichen Wesens der deutschen

Sprache zu pflegen - und

3. auf diese Weise das allgemeine nationale Bewußtsein im deutschen Volke zu kräftigen“ ( $§ 1$ der

Satzungen; Zeitschrift des ADSV, I. Jg., Nr. 1, 1886, Sp. 1; im Weiteren ZADSV I, 1, 1886, Sp. 1).

Für die Mitglieder des ADSV war die avisierte Reinigung des Deutschen eine nationale Aufgabe, wie Herman Riegel im Kapitel ,Der Kampf gegen die Fremdwörter eine nationale Pflicht' in seiner im Jahre 1887 in Dresden veröffentlichten Schrift Die Sprachreinigung und ihre Gegner betonte (vgl. Riegel 1887:75). Sie sollten sich neben der Reinigung auch der Pflege ihrer Muttersprache widmen. Im Vereinsmotto, das „Gedenke auch, wenn du die deutsche Sprache sprichst, dass du ein Deutscher bist." lautete, spiegelt sich die nationalistische Richtung wider.

Der Fremdwortpurismus war während der Existenz des ADSV stärker auf die geschriebene Sprache gerichtet (Blume 2013:15); der ADSV besaß einen eigenen Verlag, dessen Tätigkeit mit der Fremdwortbekämpfung verbunden war. Im Folgenden werden ein paar Beispiele für eine aktive Beteiligung der Repräsentanten des ADSV an der Spracharbeit dieser Art angeführt. Es wurden Verdeutschungsbücher verfasst, ${ }^{9}$ die als Ratgeber bei der außerschulischen Förderung des Sprachgebrauchs dienten, aber auch für Schule und Haus, Unteroffiziere, den öffentlichen Dienst, Hochschulen und weitere potenzielle Benutzer bestimmt waren. Das erste Verdeutschungsbuch des ADSV trägt den Titel ,Deutsche Speisekarte. Verdeutschung der in der Küche und im Gasthofswesen gebräuchlichen entbehrlichen Fremdwörter' (= Verdeutschungsbücher des Allgemeinen Deutschen Sprachvereins, Band 1). An der Entstehung der vierten Auflage dieses Werks im Jahre 1900 waren der Leiter des Dresdner Zweigvereins Hermann Dunger und der Dresdner Koch Ernst Lößnitzer beteiligt, die im ersten Kapitel ,Fremdwörterei in der Gasthofs- und Küchensprache“ auf interessante Weise ihre Bestrebungen, deutsche Äquivalente im Bereich der französischen Küche vorzuschlagen, u. a. am Beispiel einer kurzen Textprobe begründen: ${ }^{10}$

„Selbst für die gewöhnlichsten Gerichte der bürgerlichen Küche, die nicht aus Frankreich zu uns gekommen sind, wählt man französische Bezeichnungen. Es heißt nicht Eierkuchen, sondern Omelette, nicht Mus oder Brei, sondern Purée, nicht Lende, sondern Filet. Gekochtes Rindfleisch - pfui, wie gemein! Auf dem ,Menu' erscheint es als Boeuf bouilli oder naturel. Wenn man in einem Speisehaus eine Tasse Fleischbrühe bestellt, so kann man sicher darauf rechnen, daß der Kellner zuerst verdutzt sich besinnt und dann mit einem halb mitleidigen halb verächtlichen Blick erwidert: ,Ah - eine Tasse Bouillon!““( (Dunger - Lößnitzer 1900:4).

Die Zeitschrift erschien bis 1924, seit 1925 wird anstelle dieses Periodikums die Zeitschrift ,Muttersprache“ herausgegeben.

9 Albrecht Greule betont, dass die Verdeutschungsbücher nicht mit erklärenden Fremdwörterbüchern verwechselt werden dürfen (vgl. Greule 1986:30). Katarzyna Sztandarska unterscheidet drei Typen von Wörterbüchern mit Fremdwort-Lemmata: In erklärenden Fremdwörterbüchern kann der Benutzer neben grammatischen und phonetischen Informationen auch Angaben über Herkunft und Erklärung von Wörtern fremder Herkunft finden. Das Ziel von Verdeutschungswörterbüchern, in denen weder grammatische noch phonetische Angaben zur Verfügung stehen, besteht darin, fremdsprachliche Ausdrücke durch heimische Äquivalente zu ersetzen Im dritten Typ, in Fremd- und Verdeutschungswörterbüchern, d. h. in erklärend-verdeutschenden Wörterbüchern, in denen weder grammatische noch phonetische Angaben zur Verfügung stehen, werden Fremdwörter erklärt und deutsche Äquivalente für sie angeboten. Sztandarska behandelt noch Verdeutschungshefte mit der Verdeutschungsfunktion, in denen sowohl grammatische als auch phonetische Informationen fehlen (Sztandarska 2017:836-838).

10 Das Buch erschien zum ersten Mal im Jahre 1888 und bis 1900 wurden vier Auflagen veröffentlicht, was von dem großen Erfolg dieser Publikation zeugt. An der Herausgabe in den Jahren 1888, 1891 und 1897 beteiligten sich Hermann Dunger und Hofküchenmeister Friedrich Baumann (†1889). 
Die Autoren informieren im Vorwort, dass es nur in wenigen Fällen zu Neubildungen kommt, dass bei Fremdwörtern mehr als eine Verdeutschung gegeben wird und dass bei verbreiteten Fremdausdrücken neben den deutschen Ersatzwörtern fremde Formen in deutscher Schreibung erscheinen (Dunger - Lößnitzer 1900:VII-VIII), z. B. bei der verdeutschten Form Bisquit auch biscuit, bei Likör auch Liqueur, bei Bole auch Bowle. Eine kleine Lemma-Probe zeigt, dass die Autoren den Benutzern neben deutschen Äquivalenten (1) auch Informationen im Bereich der Dialektologie und Wortgeographie (2) zur Verfügung stellten:

$\begin{array}{ll}\text { (1) Asperge } & \text { Spargel. } \\ \text { Asperges à la crème } & \text { Spargel in Rahm. } \\ \text { Asperges au gratin } & \text { überkrusteter Spargel. } \\ \text { Asperges en branches } & \text { Stangenspargel. } \\ \text { Asperges frites } & \text { gebackener Spargel. } \\ \text { Asperges vertes } & \text { grüner Spargel. } \\ \text { Pointes d'asperges } & \text { Spargelköpfe, Spargelgemüse. }\end{array}$

(Dunger - Lößnitzer 1900:52)

Sabayon Weinschaum, Weinsoße, =tunke, $=g u \beta,=$ beiguß, Eierwein.

(Dunger - Lößnitzer 1900:75)

(2) Filet Lende, Rückenstück, Geflügelbrust; (längliches) Schnitzel von Fleisch, Fisch usw.

Filet de bøuf Rindslende, Lendenbraten, Mürbebraten, Lummer (Kassel, Münsteri.W.), Lummel (Posen), Marbraten (Görlitz), Lungenbraten (Österreich), Schlachtbraten (Stuttgart).

Filet mignon Lendchen (von Kalb, Wild usw.).

Filet sauté geschwungene (geschmorte) Schnitte, Schmorschnitte.

(Dunger - Lößnitzer 1900:60-61)

Manchmal zeigen sich die vorgeschlagenen Neubildungen, die die Tatsache nicht ganz zutreffend benennen, als wenig praktikabel:

$\begin{array}{ll}\text { Maccaroni } & \text { Röhrnudeln. } \\ \text { Mayonnaise } & \text { Mayonnäse,11 saurer Ölguß. } \\ \text { Nectarine } & \text { glatter (nackter) Pfirsich, Honigpfirsich } \\ \text { (Dunger - Lößnitzer 1900:66-67) }\end{array}$

Auch weitere Verdeutschungsbücher waren thematisch orientiert - das zweite Verdeutschungsbuch wurde dem Handel gewidmet, das dritte betraf die Verdeutschung der im täglichen Verkehr gebrauchten Fremdwörter, das vierte war das Namenbüchlein von Ferdinand Khull; den fünften Band ,Die Amtssprache: Verdeutschung der hauptsächlichsten im Verkehre der Gerichts- und Verwaltungsbehörden gebrauchten Fremdwörter' gab Karl Bruns heraus, der sechste umfasste das Bergund Hüttenwesen, der siebente ist der Schule gewidmet, der achte der Heilkunde und der neunte der Tonkunst, dem Bühnenwesen und Tanz. Der zehnte Band betrifft Sport und Spiel, im elften Band kam das Versicherungswesen an die Reihe, das zwölfte betrifft das deutsche Buchgewerbe, die Buchdruckerei, Schriftgießerei, Steindruckerei etc., das dreizehnte Verdeutschungsbuch bringt der Öffentlichkeit Verdeutschungen fremdsprachlicher studentischer Ausdrücke - Vorschläge und Anregungen ${ }^{12}$ und das vierzehnte Verdeutschungsbuch dient Mathematikern.

11 Vorgeschlagen wurde auch der fremde Ausdruck, in leicht verdeutschter Form.

12 Der 13. Band wurde wahrscheinlich im Jahre 1922 herausgegeben. Anke Heier merkt in ihrem Buch im Literaturverzeichnis bei diesem Titel an, dass die Information über den 13. Band der Verdeutschungsbücher des Allgemeinen deutschen Sprachvereins in einer Werbung im 14. Verdeutschungsbuch für die Größenlehre oder Mathematik im Jahr 1936 veröffentlicht wurde (vgl. Heier 2012:541). 
Die Pflege betraf, wie der vierte Band zeigt, auch Vornamen, die nach dem Motto Deutschen Kindern deutsche Namen vergeben werden sollten. Dabei konnte ein Namenverzeichnis von Ferdinand Khull behilflich sein. Das Motto war als Wunsch aller völkisch fühlenden „Volksgenossen“ zu verstehen.

Der deutsche Fremdwortpurismus, dem der Nationalismus im späten 19. Jahrhundert als Nährboden diente, war im öffentlichen Sprachgebrauch wirkungslos (Blume 2013:19). Viele vorgeschlagene Ausdrücke wie z. B. Haarkünstler, Haarschneider oder Haarkräusler für Friseur (Lohmeyer 1915:41), Raum- oder Festmeter für Kubikmeter (Lohmeyer 1915:78) oder Wärme(grad), Luftwärme für Temperatur setzten sich in der Umgangssprache nicht durch.

Universitätsgermanisten unterstützten die Tätigkeit des ADSV kaum, Ausnahmen bildeten beispielsweise Otto Behaghel oder Friedrich Kluge, die aber keine Mitglieder waren. Zu den Gegnern des Vereins gehörten z. B. der Rechtsgelehrte und Übersetzer Otto Gildemeister, der Historiker und Politiker Hans Delbrück, der Rechtsgelehrte Gustav Rümelin und der Kunsthistoriker und Romanschriftsteller Herman Grimm, der Neffe Jacob Grimms. Auf ihre Angriffe reagierte Hermann Dunger in seiner bereits erwähnten Schrift Die Sprachreinigung und ihre Gegner (Dresden 1887). Gegen die Tätigkeit des ADSV sprachen sich im Jahre 1889 in der sog. Erklärung der 41 auch 41 Persönlichkeiten aus dem universitären Gebiet und dem Kreis der Schriftsteller aus, z. B. Theodor Fontane, Gustav Freytag oder Wilhelm Dilthey, die eine Erklärung gegen diesen Verein unterschrieben. Sie waren grundsätzlich nicht gegen die Sprachreinigung, sie kritisierten aber die Tatsache, dass sich der Vorstand des ADSV mit einem Brief an den preußischen Minister der geistlichen, Unterrichts- und Medizinalangelegenheiten Dr. von Goßler mit folgender Bitte gewandt hatte:

„Euer Exzellenz wolle hochgeneigtest in einem Erlasse an die unterstehenden Schulbehörden des preußischen Staates auf die dargelegten Bestrebungen des allgemeinen deutschen Sprachvereins beistimmend hinweisen, die Ersetzung der entbehrlichen Fremdwörter durch gute deutsche Ausdrücke empfehlen, sowie auch besonders die Lehrer des Deutschen anregen, die rein lehrmäßige Behandlung der Muttersprache in der heranwachsenden Jugend zu ergänzen, damit so der deutsche Unterricht geläutert, vertieft und zu einem bedeutsamen Mittel geistiger und nationaler Erziehung ausgestattet werde“ (ZADSV II, 1, 1889, Sp. 15).

Die Initiatoren der sog. Erklärung der 41 kritisierten die Tatsache, dass die Sprachreinigung durch Vereine und staatliche Organisationen organisiert werden sollte, und vertraten die Meinung, der ADSV versuche, die Sprache ,von oben zu regeln“. ${ }^{13}$

\section{Der ADSV und seine Zweigvereine}

Im Jahre 1890 zählte der ADSV im Deutschen Reich etwa 12000 Mitglieder; etwa ein Drittel davon - 3500 - umfasste den Handels- und Gewerbestand; Mitglieder waren weiter 1400 Kaufleute, 800 Rechtsgelehrte, 550 Fabrikanten, 330 Buchhändler und Drucker. Nicht überraschend ist die Vertretung von 2500 Lehrern, davon waren 200 Hochschullehrer und über 1000 Lehrer von Gymnasien und Realgymnasien, über 1300 von Mittel-, Volks-, Mädchen- und Fachschulen (vgl. ZADSV III, 7, 1890, Sp. 100).

Adolf Hauffen ${ }^{14}$ zufolge wurden bis 1916 insgesamt 330 Zweigvereine gegründet, im Jahre 1916 zählt der Gesamtverein an die 37000 Mitglieder - nicht nur im In-, sondern auch im Ausland, im

13 In dieser Studie geht es nicht darum, die ganze Geschichte des ADSV zu präsentieren, sondern nur einen historischen Rahmen für die Tätigkeit der Zweigvereine in Mähren zu schaffen. Abschließend ist noch zu bemerken, dass der ab 1923 Deutscher Sprachverein genannte ADSV eine Blütezeit während des I. Weltkrieg und in der Weimarer Republik erlebte; im Jahre 1947 wurde die Gesellschaft für deutsche Sprache gegründet, ihre Zeitschrift Muttersprache wird bis heute herausgegeben.

14 Adolf Hauffen wurde 1894 zum Obmann des Zweigvereins in Prag gewählt (ZADSV IX, 5, 1894, Sp. 103). Er studierte Deutsch, Geschichte und Geographie an der Universität in Wien, Deutsch und Englisch in Leipzig, Deutsch in Graz und Berlin. Er war an der deutschen Universität in Prag als Privatdozent (1889-1896), außerordentlicher Professor 
italienischen Teil der Schweiz, in Belgien, Luxemburg und in anderen Ländern, so z. B. eben auch in Böhmen und Mähren. Jeder Zweigverein erhielt 25 Satzungen des ADSV, in denen auch Satzungen für die Tätigkeit der Zweigvereine erfasst wurden (Landesarchiv Brünn, B14 ml., Kartonnummer 51). In Österreich entstanden nach Hauffens Angaben 32 Zweigvereine mit über 3200 Mitgliedern; der erste Zweigverein in Österreich wurde am 4. September 1886 in Krems an der Donau gegründet (Hauffen 1916/17: 300). In Böhmen entstanden insgesamt 15 Zweigvereine, und zwar in TeplitzSchönau (Teplice), ${ }^{15}$ Reichenberg (Liberec) ${ }^{16}$ Karlsbad (Karlovy Vary), Eger (Cheb), ${ }^{17}$ Leipa (Česká Lípa), ${ }_{18}^{18}$ Prag (Praha), ${ }^{19}$ Gablonz (Jablonec), Budweis (Budějovice) ${ }^{20}$ Leitmeritz (Litoměřice), Haida (Nový Bor), ${ }^{21}$ Komotau (Chomutov), Aussig (Ústí nad Labem), Franzensbad (Františkovy Lázně), Teschen-Bodenbach (Děčín) und Obersedlitz-Krammel (Novosedlice-Kramoly). In Gablonz hat sich ein bereits bestehender älterer Sprachverein, der etwa 150 Mitglieder zählte, dem ADSV als Zweigverein angeschlossen (ZADSV III, 5, 1888, Sp. 77). Der stärkste Zweigverein in Böhmen, in Reichenberg, wurde im Jahre 1887 gegründet und entwickelte eine äußerst aktive Vortragstätigkeit. Sein Schriftleiter Karl Sedlak wurde im Jahre 1889 zum Mitglied des Gesamtvorstands des ADSV ernannt, und seitdem hatten die böhmischen Zweigvereine in diesem Kollegium ihre Vertretung (ZADSV IV, 6, 1889, Sp. 101). Der ADSV verfolgte aufmerksam die Tätigkeit der Zweigvereine, auch derjenigen in den böhmischen Ländern, und brachte darüber regelmäßige Informationen auf Seiten seiner Zeitschrift, die als gutes Beispiel für andere Zweigvereine dienen sollten. So hat beispielsweise der Vorstand des Teplitzer Zweigvereins im Jahre 1890 beschlossen, Sprachabende zu veranstalten, ,an denen volkstümliche Vorträge abgehalten werden sollten, um so der Bevölkerung von Teplitz und Umgebung Gelegenheit zu bieten, sich von den Bestrebungen und Zielen des Allgemeinen deutschen Sprachvereins persönlich zu überzeugen“ (ZADSV VI, 5, 1891, Sp. 74). Gehalten wurden Vorträge über die Mundarten, Volksüberlieferungen und Dichter, die in der Region lebten; Alexander Trag $1^{22}$ hielt in Leipa einen Vortrag über dortige Ortsnamen, Josef Just einen Vortrag über heimische mundartliche Ausdrücke (Hauffen 1916/17: 350), Michael Mottal in Teplitz präsentierte einen Vortrag über „die Entstehung des Fremdwörterunfugs in der deutschen Sprache“ (ZADSV VI, 5, 1891, Sp. 74), ähnlich war die Lage in Leitmeritz, wo der Schriftführer des Zweigvereins Hähnel einen Votrag ,über die Lehnwörter und Fremdwörter im Deutschen“ hielt (ZADSV V, 6, 1890, Sp. 93). Das folgende Beispiel kann belegen, dass der Gesamtvorstand des ADSV der Tätigkeit der Zweigvereine sorgsame Aufmerksamkeit widmete: Im Jahre 1891 wurde auf Seiten der Zeitschrift des ADSV der Schriftführer des Zweigvereins in Teplitz Michael Mottal für seine unermüdliche Tätigkeit für die Vereinssache mit der Begründung gelobt, er habe in der Zeitung „Teplitz-Schönauer Anzeiger seine Stimme über ,Sprachreinigung' erhoben und einen Ruf an die Bevölkerung Redet deutsch! ergehen lassen," was als besonders empfehlenswertes Exempel dargestellt wurde (ZADSV VI, 6, 1891, Sp. 94).

(ab 1896) und ordentlicher Professor für Deutsche Sprache und Literatur (1919-1930) tätig. Im Jahre 1894 wurde er Obmann des Zweigvereins in Prag, diesen Posten bekleidete er bis 1898 (Vodrážková 2007:104), später auch in den Jahren 1900-1923. Weitere Mitglieder des Prager Zweigvereins waren seit 1888 Hans Lambel und August Sauer.

15 Der Zweigverein wurde - so die Zeitschrift des ADSV - relativ früh, im Jahre 1886, unter Führung Herman Frohnes gegründet (ZADSV I, 4, 1886, Sp. 67).

16 Über die Gründung des Zweigvereins des ADSV in Reichenberg informiert die Zeitschrift des ADSV in der Nummer vom 1. 2. 1887 (ZADSV I, 9, 1887, Sp. 147).

17 Die Zeitschrift des ADSV meldet die Gründung der Zweigvereine in Eger und Karlsbad im Jahre 1888 (ZADSV III, 2, 1888, Sp. 29).

18 Die Information über die Gründung des Zweigvereins brachte die Zeitschrift des ADSV im April 1888, bei der Gründung anwesend war Karl Sedlak aus Reichenberg (ZADSV III, 4, 1888, Sp. 63).

19 Die Zeitschrift des ADSV brachte einen kurzen Bericht über den Zweigverein in Prag am 1. 12. 1887, an der Spitze des Zweigvereins stand Dr. Sedlak aus Reichenberg (ZADSV I, 17, 1887, Sp. 287).

20 Vgl. die Information vom 1. 5. 1888 (ZADSV III, 5, 1888, Sp. 77).

21 Vgl. die Information vom 1. 10. 1889 (ZADSV IV, 10, 1889, Sp. 167).

22 Alexander Tragl war Schuldirektor des Gymnasiums in Böhmisch Leipa, später in Kaaden. Er gab im Jahre 1896 die Studie über die ,Leipaer Familiennamen“ im Jahresbericht des k. k. Staats-Ober-Gymnasiums in Böhmisch Leipa heraus. 
Die ausgewählten Vertreter von Zweigvereinen hatten wahrscheinlich die Möglichkeit, jedes Jahr an der Tagung der Hauptversammlung des $\mathrm{ADSV}^{23}$ teilzunehmen. Mähren war nie vertreten, wovon beispielsweise die siebente Hauptversammlung des ADSV im August 1894 zeugt, in der unter den anwesenden Vertretern der Zweigvereine keiner aus Mähren war. ${ }^{24}$ Nur selten anwesend waren die böhmischen Zweigvereine; Belege stammen aus Leipa, Leitmeritz, Prag und Reichenberg, so erfahren wir, dass in der siebenten Hauptversammlung Professor Menzl den Zweigverein Reichenberg und der Postrat Dr. Dehms aus Potsdam den Zweigverein Teplitz vertrat (ZADSV IX, 9, 1894, Sp. 171-172).

Es ist nicht das Ziel dieser Studie, die Tätigkeit der böhmischen Zweigvereine detailliert vorzustellen. ${ }^{25}$ Eine kurze Charakteristik wurde in dieser Studie deswegen vorgelegt, um eine Basis für eine nähere Beschreibung der Tätigkeit in den mährischen Zweigvereinen zu schaffen. Aus diesen kurzen Informationen ergibt sich, dass manche böhmischen Zweigvereine vor allem in der Vortragstätigkeit aktiv waren, die sich auf den Fremdwortpurismus und auf die Präsentation der deutschen Kultur in der betreffenden Region, auch beispielsweise der Mundarten, konzentrierte.

\subsection{Die Zweigvereine des ADSV in Mähren}

In Mähren führt Adolf Hauffen drei Städte an, in denen Zweigvereine gegründet wurden - Neutitschein (Nový Jičín), Brünn (Brno) und Iglau (Jihlava). Bei Neutitschein findet sich die Bemerkung, dass der Zweigverein bald wieder eingegangen sei, bei Brünn die Information, dass ,die gründende Versammlung vom 24. Februar 1893 gleichzeitig das Ende dieses Zweigs bedeutete“, wobei Hauffen das Bedauern ausdrückte, dass „,die Hauptstadt Mährens, deren Stadtvertretung deutsch ist, es bis heute zu keinem Zweigverein gebracht hat" (vgl. Hauffen 1916/17:300). Da in der Fachliteratur nichts Näheres zu einer weiteren Entwicklung dieser mährischen Zweigvereine gefunden wurde, war es notwendig, eine Untersuchung mit dem Bestreben zu führen, die Informationen Hauffens zu präzisieren und eventuell zu ergänzen. Es gibt nur wenige Archivquellen zu diesem Thema - ein bescheidener Bestand wird im Landesarchiv Brünn aufbewahrt; der Bestand im Iglauer Staatlichen Bezirksarchiv ist noch geringer, und aus dem Staatlichen Bezirksarchiv Neutitschein kam eine negative Antwort in dem Sinne, es gebe keinen selbstständigen Bestand, nur vereinzelte Informationen über den Zweigverein des ADSV in Neutitschein. Die erhaltenen Belege in den drei genannten Archiven sind zwar bescheiden, trotzdem wird ein Versuch gemacht, wenigstens einige Personen vorzustellen, die in diesen Zweigvereinen tätig waren, und wenn es die Quellen erlauben, auf ein paar Momente in der Tätigkeit der Zweigvereine hinzuweisen.

Als Beleg für ein bilinguales Milieu in diesen drei mährischen Städten wird am Anfang die Anzahl der Deutschen und/oder der Tschechen im Jahre 1880 angegeben. Die Stadt Brünn zählte 1880 insgesamt 109339 Einwohner, davon 48591 Deutsche (HSBM 1998:74); in Iglau lebten 19269 Deutsche und 3715 Tschechen (HSBM 1998:219) und Neutitschein wies insgesamt 10274 Einwohner auf, davon 1095 Tschechen (HSBM 1998:409).

Die bescheidensten Informationen finden wie bereits angeführt für den Zweigverein in Neutitschein. Die Zeitschrift des ADSV brachte am 1. August 1889 in der Rubrik „Aus den Zweigvereinen“ die Nachricht, dass bei einem in Neutitschein veranstalteten Sängerfest 23 neue Mitglieder für den Verein gewonnen werden könnte, so dass der Zweigverein in dieser Stadt 112 Mitglieder zählte (ZADSV IV, 8/9, 1889, Sp. 149). Hauptmitglieder des Zweigvereins waren der Obmann Johann Tuschina, Direktor der Neutitscheiner Realschule, der Obmann-Stellvertreter Rainer Hosch, Buchhändler und Verleger in Neutitschein; Schriftwart war Karl Peschke, Lehrer und Kustod des im Jahre 1889 eröffneten Neutitscheiner Museums, Zahlmeister Dr. Johann Berthold Wolf, Vizepräsident

23 Die Hauptversammlungen fanden jedes Jahr in einem anderen deutschen Ort statt, z. B. 1887 in Dresden, 1888 in Kassel, 1894 in Koblenz usw.

24 Vertreten waren die böhmischen Zweigvereine Eger, Leipa, Leitmeritz, Prag, Reichenberg (ZADSV IX, 9, 1894, Sp. 171-172).

25 Diese Aufgabe wird Eva Janečková in einem selbstständigen Aufsatz übernehmen, der vorbereitet wird. 
des Kreisgerichts in Neutitschein, und Ausschussmitglieder waren Friedrich Hirth, ein Lehrer an der dortigen Realschule und der erste Kustod im Neutitscheiner Museum, ${ }^{26}$ Dr. Florian Schimann, in den Jahren 1900-1901 Bürgermeister in Neutitschein, Rudolf Weiß d. Ä., Inhaber der Tuch- und Wollwarenerzeugung Franz Jakob Weihs Sohn, der auch Mitglied des Männergesangvereins ${ }^{27}$ in Neutitschein war; in den Jahren 1887-1897 stand er an der Spitze dieses Chors und in den Jahren 1870-1909 war er Mitglied des Stadtrates. Derzeit gibt es keine weiteren Informationen mehr über die Tätigkeit des Neutitscheiner Zweigvereins. Daher ist nur zu konstatieren, dass der Zweigverein wahrscheinlich keine großen Aktivitäten auf dem Gebiet des Fremdwortpurismus entwickelte; vielmehr konzentrieren sich die Mitglieder des Ausschusses auf eine heimatkundliche Arbeit, indem sie sich beispielsweise an der Tätigkeit des Museums aktiv beteiligten, um „das allgemeine nationale Bewußtsein im deutschen Volke zu kräftigen“ (§ 1 der Satzungen, ZADSV I, 1, 1886, Sp. 1). Diese Aktivität war aber keine spezielle Aufgabe dieses Sprachvereins, sondern sie erscheint in der Tätigkeit auch anderer regionaler deutscher Vereine im Lande. Das Bestehen des Zweigvereins in Neutitschein dauerte bis 1894; in diesem Jahr wurde er aufgelöst (ZADSV X, 2, 1895, Sp. 37).

Ein kleines Rätsel stellt der Zweigverein in der mährischen Stadt Brünn dar. Die Information über die Gründung der dortigen Organisation wurde in der Zeitschrift des ADSV am 1. Juni 1890 mit der Anmerkung veröffentlicht, dass dies auf Anregungen eines gewissen Prof. Habermann von der technischen Hochschule geschah (ZADSV V, 7, 1890, Sp. 116). Diese Tatsache wurde auch noch an einer anderen Stelle in dieser Zeitschrift, im „Geschäftlichen Teil“, abgedruckt, wo betont wurde, dass es zur Gründung durch Professor Habermann von der technischen Hochschule auf Veranlassung von Dr. Thießen aus Berlin kam (ZADSV V, 7, 1890, Sp. 115), was die Vorstellung evoziert, Berlin hätte großes Interesse an der Gründung eines Zweigvereins in der Hauptstadt Mährens gehabt. Dass dieser Versuch nicht erfolgreich war, belegt eine Nachricht in der Zeitschrift des ADSV vom 1. April 1893. Wir erfahren, dass die begründende Versammlung eines neuen Zweigvereins am 24. Februar 1892 stattfand; auf diesem Treffen legte Dr. Gustav Gottlieb Trautenberger einen Überblick über die Entstehungsgeschichte des Zweigvereins vor; später zeigte sich, dass sich sowohl der frühere als auch dieser zweite Zweigverein nicht als lebensfähig erwiesen. Nach diesen zwei erfolglosen Versuchen kam es erst im Anschluss an einen Vortrag über die deutsche Sprache, der vom Geschichtslehrer im Brünner Untergymnasium Julius Miklan abgehalten wurde, zu einer vorbereitenden Versammlung am 27. Mai 1892, die nach der Genehmigung der Satzungen endlich zur Gründung des Zweigvereins führte (ZADSV VII, 4, 1893, Sp. 61). Es hat auch weiterhin unausgesetzter Bemühungen bedurft, um in dieser Stadt den Zweigverein am Leben zu halten, wobei angemerkt wird ,ähnlich wie in der Stadt Konstanz“, so die Zeitschrift des ADSV (VII, 4, 1893, Sp. 70). Am 5. Oktober 1892 legten Dr. Gustav Trautenberger Senior und Dr. Stefan Licht als Vertreter des Vorbereitungsausschusses die Satzungen des Zweigvereins zur Bescheinigung vor; am 4. November 1892 genehmigte das Ministerium des Innern die Bildung des Zweigvereins Brünn und Umgebung nach Inhalt der Statuten und den Anschluss dieses Vereins an den Allgemeinen deutschen Sprachverein. Erst am 24. Februar 1893 wurde der Brünner Zweigverein konstituiert (LAB, B14 ml., Kartonnummer 51, Urkundennummer 7.730/1893). Dieser Brünner Fall zeigt, wie kompliziert es war, in der habsburgischen Monarchie einen Verein zu gründen. Das österreichische Vereinsgesetz enthielt manche komplizierte Bestimmung, beispielsweise die einzuholende Genehmigung des k. k. Ministeriums des Innern (vgl. ZADSV I, 5, 1886, 74). In Anbetracht dieses Aufwandes ist es so überraschender, dass der Brünner Zweigverein seit 1894 keine Tätigkeit mehr entwickelte; die Jahresversammlung konnte nicht mehr abgehalten werden, so Josef Matzura, der k. k. Professor und Schatzmeister des Vereins. ${ }^{28}$ Am 3. März 1897 löste sich der Brünner Zweigverein infolge Mangels

26 Fritz Hirth war Autor des populären Liederbuchs für deutsche Studenten, um 1890 wurde die 11. Auflage herausgegeben, und des Liederbuchs für deutsche Studenten und Turner. Er war auch Gauobmann des mährisch-schlesischen Turngaus (vgl. http://www.znaim.eu/8-Vereine/Bruenn und die voelkische Turnbewegung [13.03.2021]).

27 Männergesangvereine wurden vor allem in der Zeit ab 1830 gegründet. Der Gesang brachte patriotische, nationale und freiheitliche Texte leichter zu Gehör; sie konnten zur politischen Meinungsbildung benutzt werden (Polenz 1999:66).

28 Das Protokoll über die freiwilligen Auflösung des Brünner Zweigvereins wurde am 13. Februar 1897 an der k. k. Polizei- 
an Mitgliedern freiwillig auf(LAB, B14 ml., Urkundennummer 9.939/1897). Diesen kurzen Bericht veröffentlichte am 10. März 1897 die Brünner Zeitung (vgl. Brünner Zeitung 56, 10. 3. 1897, 4).

Was wissen wir von den Brünner Hauptvertretern des Zweigvereins? Dr. phil. et Lic. theol. Gustav Gottlieb Trautenberger (1836-1902) war ein evangelischer Geistlicher, Senior des mährischen Seniorrats, Kirchenhistoriker und eine bedeutende Persönlichkeit der deutschen Kultur im Brünn der 2. Hälfte des 19. Jahrhunderts. Er erwarb die akademischen Grade an den Universitäten Tübingen und Wien. Dr. Stefan von Licht (1860-1932), Politiker der Deutschen Nationalpartei, war als Rechtsanwalt in Brünn tätig (vgl. Österreichisches biographisches Lexikon 1972, 184). Es waren ähnlich wie in Neutitschein - Repräsentanten des öffentlichen Lebens, der eine war Rechtsanwalt und der andere ein evangelischer Geistlicher, keiner von ihnen war Linguist und Fremdwortkämpfer. Germanistik und Geschichte studiert hatte an der Wiener Universität der dritte Mann des Brünner Zweigvereins, Josef Matzura (1851-1936), der an der deutschen Staatsgewerbeschule in Brünn tätig war. Aber auch er konzentrierte sich eher auf eine heimatkundliche Tätigkeit und verfasste im Jahre 1891 einen illustrierten Führer durch die Beskiden (vgl. Österreichisches biographisches Lexikon 1815-1950, Bd. 6, 155). Für die Fremdwortbekämpfung interessierte er sich gar nicht.

Der dritte mährische Zweigverein des ADSV entstand in Iglau. Am 25. Juni 1893 wendeten sich Robert Honsig, Josef Vorreiter und Carl Langer als Mitglieder des Gründungsausschusses mit einem Brief an die k. k. mährische Statthalterei mit dem Antrag, die vorgelegten Statuten eines Zweigvereins des Allgemeinen deutschen Sprachvereins, die nach dem Vorbild der Statuten des Hauptvereins verfasst wurden, zu genehmigen. Im Juli 1893 kam eine abschlägige Antwort von der k. k. mährischen Statthalterei in Brünn nach Iglau mit der Begründung, der Zweigverein müsse zuerst gegründet werden und erst danach könnten die Satzungen bestätigt werden. Aus diesem Grund verfassten die Mitglieder des Gründungsausschusses ein neues Gesuch, das mit sieben Beilagen an das k. k. Ministerium des Innern / die k. k. Statthalterei nach Brünn abgeschickt wurde:

\begin{abstract}
„Die ergebenst Gefertigten gedenken in Iglau in Mähren einen Zweigverein des allgemeinen deutschen Sprachvereines zu gründen, legen zu diesem Behufe den Satzungsentwurf dieses zu gründenden Zweigvereines vor und stellen die ergebenste Bitte:

Das Hohe k. k. Ministerium des Inneren geruhe die Gründung des Zweigvereines Iglau des allgemeinen deutschen Sprachvereines zu bewilligen. Den Rechtsbestand des allgemeinen deutschen Sprachvereines bescheinigen die ergebenst Gefertigten durch Vorlage der Satzungen dieses Vereines, welchem eine Übersicht der in Österreich bereits befindlichen Zweigvereine beigeschlossen ist, aus welcher erhellt, daß auch in Mähren, und zwar in Neu-Titschein ein Zweigverein dieses Vereines, sowie mehrere Zweigvereine in den anderen Kronländern der öster.-ung. Monarchie bestehen.“ Iglau, am ... ${ }^{29}$ Juli 1893
\end{abstract}

Josef Vorreiter, Schriftleiter

Robert Honsig, Oberlehrer Carl Langer

Die Gründung des Zweigvereins und die Satzungen wurden laut Erlass des k. k. Ministeriums des Innern vom 17. August 1893 (Zahl 19846) genehmigt (Zuschrift des ersten Gemeinderates der königlichen Stadt Iglau vom 14. 9. 1893, Zahl 10751/pol. - Iglau, Archiv). ${ }^{30}$ Das Motto oder der Leitspruch des Iglauer Zweigvereins war Kein Fremdwort für das, was gut deutsch ausgedrückt werden

direktion Brünn aufgenommen, bei dieser Gelegenheit erklärte der Säckelwart des Zweigvereins Professor an der k. k. deutschen Gewerbeschule Josef Matzura, dass der Zweigverein seit 1894 keine Tätigkeit infolge Mangels an Mitgliedern mehr entwickelte und die Jahresversammlung nicht stattfinden konnte. Die Worte von Josef Matzura belegt ein Schreiben vom 27. Oktober 1894 von Julius Miklan, dem k. k. Professor, an die k. k. Polizeidirektion. Miklan informiert über den Rücktritt des Obmanns Ignaz Pokorny, des Regierungs- und Schulrats, über die Tatsache, dass auch er - Miklan sich zurückzog, und empfiehlt der Direktion, sich mit der Frage, ob der Brünner Zweigverein noch bestehen wird, an Josef Matzura zu wenden (LAB, B26, Kartonnummer 2460, Sign. 97).

29 Eine leere Stelle für das aktuelle Datum wurde nicht ausgefüllt. Wahrscheinlich wurde der Brief am 30. 7. verfasst, am 31.7.1893 wurde er zugestellt (LAB Brno, B26, Kartonnummer 2460, Sign. 97).

30 Über die Gründung des Zweigvereins in Iglau informierte auch die Zeitschrift des ADSV (vgl. ZADSV IX, 9, 1894, Sp. 163). 
kann (Deutscher Volkskalender für die Iglauer Sprachinsel 10, 1896, 146). Der Zweigverein, der die offizielle Bezeichnung Zweigverein zu Iglau und Umgebung in Mähren trug, hatte im Jahre 189320 und im Jahre 189423 Mitglieder (ZADSV IX, 12, Sp. 225). Könnte uns die Frage „Wer waren die Iglauer Männer an der Spitze der Organisation?“ etwas über die Tätigkeit des Iglauer Zweigvereins sagen? Robert Honsik (1840-1924) ${ }^{31}$ war Oberlehrer an der Knabenvolksschule in Iglau und neben einem Posten im Zweigverein bekleidete er auch den des Vorstandes der Iglauer Ortsgruppe des Deutschen Schulvereins. ${ }^{32}$ Josef Vorreiter (1848-1920) war Professor am Iglauer Staatsgymnasium, durch die Heirat mit Hermine Rippl wurde er Mitglied der bekanntesten Iglauer Druckerfamilie; sein Schwiegervater gab die Zeitung ,Mährischer Grenzbote' heraus und Josef Vorreiter war 41 Jahre lang als deren Redakteur tätig (Knápková 2010:28-29, 451). Carl Langer (1828-1911) war Stadtverordneter und Mitglied des Bezirkschulrates. ${ }^{33}$ Im Jahre 1896 war eines der Mitglieder auch Josef Trübwasser (Deutscher Kalender 1896:146), der Dramatiker und Schriftsteller (1867-1902) war und einen Artikel über deutschen Vornamen verfasste (Unsere Vornamen. In: Deutsche Wacht, 27. 4. 1903, 1-3), daneben auch kleine literarische Texte, aber keine linguistischen Arbeiten (Knápková 2010:443). Diese Angaben zeigen, dass eher eine heimatkundliche Arbeit auf dem Gebiet der Kultur den Schwerpunkt der Tätigkeit des Zweigvereins bildete, kaum eine sprachpuristische.

Unter den sporadischen Belegen für die Tätigkeit des Iglauer Zweigvereins werden im Iglauer Archiv die offiziellen Einladungen zur Jahresversammlung in den Jahren 1903-1920 aufbewahrt, die an den Gemeinderat der königlichen Stadt Iglau geschickt wurden. Das avisierte Programm der Jahresversammlung, die in der Iglauer Sängerhalle stattfand, war immer gleich: Tätigkeitsbericht, „Geldgebarungsbericht“, Wahl des Ausschusses und Freie Anträge. Es gab wahrscheinlich Probleme mit der Anzahl der Teilnehmer an diesen Jahrestreffen, was folgender Nachtrag in der Einladung vom Jahre 1905, wonach die Jahresversammlung um 19 Uhr eröffnet werden sollte, andeutet: „Sollte die Versammlung um 7 Uhr nicht beschlußfähig sein, findet dieselbe um 8 Uhr ohne Rücksicht auf die Zahl der anwesenden Mitglieder, statt" (StBA Iglau, AMJ, 1755).

Nur ein Beleg zeigt etwas aus der Tätigkeit der Ortgruppe. Es ist ein Beschluss vom 13. 12. 1916, mit dem die „Abhaltung einer auf Vereinsmitglieder beschränkten Versammlung des Deutschen Böhmerwaldbundes und des Allgemeinen deutschen Sprachvereines für den 18. Mai in der Sängerhalle bewilligt" wurde. Diese Tätigkeit betraf zwar nicht die Sprachreinigung, aber ihr Ziel war eine „Kräftigung des allgemeinen nationalen Bewusstseins im deutschen Volke“ (StBA Iglau, AMJ, 1755).

Während der ganzen Tätigkeit des Iglauer Zweigvereins blieb Robert Honsig an der Spitze und wurde in den Akten als Vorsitzender, Obmann oder erster Sprachwart, im letzten Brief an den Stadtrat zudem als Schuldirektor in Rente bezeichnet. In diesem letzten Brief vom 3. Mai 1920 informierte Honsig über die Auflösung des Zweigvereins (StBA Iglau, AMJ, 1755).

An drei Beispielen wurde versucht, die Geschichte der mährischen Zweigvereine des ADSV zu skizzieren. Da die Belege wenig ergiebig sind, war eine ausführlichere Beschreibung der Tätigkeit nicht möglich. Aus dem bescheidenen Überblick ergibt sich der Schluss, dass die Zweigvereine des ADSV in Mähren eher am Rande des städtischen Vereinswesens standen. Die Ausschussmitglieder waren geehrte Bürger mit einer geschätzten sozialen Stellung - Lehrer an Mittelschulen, Juristen, evangelische Würdenträger, die sich aber eher auf die heimatkundliche Tätigkeit konzentrierten. Es stellt sich noch die Frage, wie viele Mitglieder eigentlich die Zweigvereine in Mähren hatten, wieviel Prozent der Einwohner deutscher Abstammung Mitglieder der Zweigvereine im Vergleich mit anderen Zweigvereinen im Land und außerhalb der böhmischen Länder waren. ${ }^{34}$

\footnotetext{
Vgl. https://www.jihlava.cz/honsig-robert/d-498703 [10.03.2021].

Vgl. https://www.jihlava.cz/honsig-robert/d-498703 [10.03.2021].

Vgl. https://www.jihlava.cz/langer-karl/d-498743 [10.03.2021].

Die Angaben entstammen der Zeitschrift des ADSV (IX, 12, 1894, Sp. 225-226).
} 


\begin{tabular}{|l|l|l|l|}
\hline Stadt & $\begin{array}{l}\text { Einwohnerzahl } \\
\text { in Tausenden (1894) }\end{array}$ & $\begin{array}{l}\text { Mitgliederzahl } \\
\text { des ADSV (1894) }\end{array}$ & \% \\
\hline Berlin & 1578,7 & 80 & 0,005 \\
\hline Braunschweig & 101 & 245 & 0,242 \\
\hline Dresden & 276,5 & 270 & 0,098 \\
\hline Frankfurt am Main & 179,9 & 160 & 0,089 \\
\hline Münster & 49,3 & 174 & 0,353 \\
\hline Innsbruck & 23,3 & 175 & 0,751 \\
\hline Wien & 1365 & 91 & 0,007 \\
\hline Budweis & 28,4 & 40 & 0,140 \\
\hline Eger & 18,6 & 50 & 0,269 \\
\hline Prag & 183 & 67 & 0,037 \\
\hline Reichenberg in Böhmen & 31 & 175 & 0,565 \\
\hline Teplitz & 18 & 92 & 0,511 \\
\hline Gablonz an d. N. & 14,6 & 90 & 0,616 \\
\hline Leipa & 10,4 & 38 & 0,365 \\
\hline Leitmeritz & 11,3 & 39 & 0,345 \\
\hline Brünn & Keine Angaben im Jahre 1894 vorhanden. & \\
\hline Iglau & $\mathbf{2 3 , 7}$ & $\mathbf{2 3}$ & $\mathbf{0 , 0 9 7}$ \\
\hline Neutitschein & $\mathbf{1 0 , 2 ^ { 3 5 }}$ & $\mathbf{0 , 3 2 3}$ \\
\hline
\end{tabular}

Tab. 1: Mitgliederzahl des ADSV im Vergleich zur Einwohnerzahl in ausgewählten deutschen, böhmischen und mährischen Städten

In Iglau war eine ähnliche Vertretung der Einwohner in Prozenten wie in Dresden - in Iglau $0,097 \%$, in Dresden 0,098 \%; in Neutitschein registrieren wir eine ähnliche Vertretung wie in Münster (Neutitschein 0,323 \%, in Münster 0,353 \%). Die Angaben in Iglau und Neutitschein sind, was die Vertretung der deutschen Bevölkerung in Zweigvereinen des ADSV betrifft, mit manchen Städten im Deutschen Reich vergleichbar. Es stellt sich die Frage, warum die Zweigvereine nur in drei mährischen Städten und nicht in anderen Städten Mährens mit deutscher Bevölkerung gegründet wurden, beispielsweise in Olmütz, wo 6123 Tschechen und 12879 Deutsche im Jahre 1880 und 6194 Tschechen und 12664 Deutsche im Jahre 1890 lebten (Nešpor 1936:261). Ist an ungenügendem Interesse der Deutschen in den mährischen Städten, einen solchen Zweigverein zu gründen, eine reiche Tätigkeit anderer Vereine auf dem Territorium der Stadt schuld? Beispielsweise waren in Olmütz im Jahre 1886 insgesamt 67 und im Jahre 1887 insgesamt 70 Vereine tätig, unter anderen der Männergesangverein, Musikverein, Verein der Deutschen in Mähren, Geschworenenverein, Ortsgruppe Olmütz und Umgebung des deutschen Schulvereins, Verein zur Stiftung eines deutschen Vereinshauses, Club der deutschen Lehrer in Olmütz, Frauen- und Mädchenortsgruppe des deutschen Schulvereins, Verein deutscher Lehrer und Schulfreunde des Landbezirks Olmütz, Deutscher Schulkreuzer-Verein, Bundesgruppe Olmütz des Bundes der Deutschen Nordmährens und weitere mehr (Statistisches Jahrbuch der königlichen Hauptstadt Olmütz 1888:507 ff.). Daraus resultieren wahrscheinlich zersplitterte Interessen der Deutschen.

In Iglau war die Anzahl der Vereine niedriger; auf dem städtischen Territorium und in der Umgebung entwickelten die Tätigkeit z. B. der Deutsche Lehrerverein F. A. Diesterweg, Deutscher Böhmerwaldbund, Deutscher Schulverein, Frauen- und Mädchenortsgruppe, Deutscher Schulverein, Ortsgruppe Nr. 105 und andere. Diese Tatsache könnte auch eine Rolle spielen. 


\section{Schlussfolgerungen}

Bereits ein paar Blicke in die Zeitschrift des ADSV - in die Rubrik „Aus den Zweigvereinen“ zeigen, dass die Zweigvereine auf dem Territorium des Deutschen Reichs und auch einige Zweigvereine in Böhmen eine reichhaltige Vortragstätigkeit organisierten, z. B. wurde in Gera im Jahre 1898 ein Vortrag zum Thema ,Schriftsprache und Volksmundart', in Grimma einer zum Thema Das Fremdwortübel und in Kassel zum Thema ,Einführung einer deutschen Speisekarte‘ gehalten (vgl. ZADSV XIII, 10, 1898, Sp. 204), im Jahre 1899 in Berlin-Chalottenburg ein Vortrag ,Das Fremdwort in der Sprache des Heeres' und in Chemnitz der Vortrag des Verlagsbuchhändlers Voigtländer aus Leipzig über ,Fremdworttorheiten“ (ZADSV XIV, 5, 1899, Sp. 105). Aus Reichenberg kam eine interessante Nachricht im Juli 1898: „Um das Vorgehen des A. D. Sprachvereins bezüglich der Gasthofsprache zu unterstützen, hat der Zweigverein Anschreiben an Gasthofbesitzer drucken lassen, die eine Liste der in der Küchensprache entbehrlichen Fremdwörter enthalten“ (ZADSV XIII, 7, 1898, Sp. 141). Eva Janečková zufolge entwickelte sich in Reichenberg eine interessante Vortragstätigkeit, beispielsweise wurden die Vorträge ,Das Fremdwort im deutschen Hause“ im Jahre 1887 von Karl Sedlak, ,Geschichte der Sprachreinigung، im Jahre 1897 von Anton Bielau und Kaufmannsdeutsch im Jahre 1907 von Arthur Ziegler gehalten (vgl. Janečková 2021, im Druck). Solche Informationen über die Vortragstätigkeit der Zweigvereine in den drei mährischen Städten wurden nicht gefunden. Wahrscheinlich entwickelten die Zweigvereine in Böhmen eine intensive Tätigkeit infolge eines größeren Einflusses des Deutschen Reichs, das in der Nachbarschaft lag. Reichenberg befand sich fast an der Grenze zum Deutschen Reich, während die drei mährischen Städte in der Mitte Mährens lagen. Eine Rolle konnte auch ein größerer Einfluss des österreichischen Deutsch in Mähren spielen, so dass ein direkter Einfluss des ADSV in Mähren nicht so groß war. Die geringe Aktivität der Zweigvereine bleibt eine Episode am Rande der Geschichte der Deutschen in Mähren.

Bereits im Jahre 1886 veröffentlichte die Zeitschrift des ADSV einen interessanten Beitrag von Aurelius Polzer aus Horn in Niederösterreich, der darüber nachdachte, warum es für Österreich kaum von Interesse ist, die Gründung von Zweigvereinen zu unterstützen. Unter anderen führt er an:

„Ich besorge nun, daß durch die Gründung von Zweigvereinen des Allgemeinen deutschen Sprachvereins unsere Kräfte zersplittert und unsere anderen nationalen Vereine geschädigt werden könnten. Und in diesen schon bestehenden Vereinen [...] liegt doch der Schwerpunkt unserer Vertheidigung gegen die Gegner der deutschen Nation. [...]. [W]ir hätten in Österreich viel wichtigere Dinge auf dem Felde des nationalen Kampfes zu thun, als unsere Sprache zu reinigen und zu retten“" (ZADSV I, 5, 1886, Sp. 74-75).

Es scheint, dass diese Gründe auch in Mähren das größte Hindernis auf dem Weg zur Gründung von Zweigvereinen waren. Wenn es gelang, einen solchen Verein zu gründen, war die Aktivität der Mitglieder zu gering oder es gab sogar überhaupt kein Interesse, sich aktiv an der Vereinstätigkeit zu beteiligen.

\section{Literaturverzeichnis}

\section{Primärliteratur:}

Brünner Zeitung Nr. 56, 1897, 10. 03.

MLB = Mährisches Landesarchiv Brno, Bestand B14 ml., Kartonnummer 51, Urkundennummer 7.730/1893; Kartonnummer 72, Urkundennummer 9.939/1897; Bestand B26, Kartonnummer 2460, Sign. 97.

StBA Iglau $=$ Staatliches Bezirksarchiv Iglau, Bestand Archiv der Stadt Iglau (AMJ), Pol. Reg. 1891-1923, Inventarnummer 1755.

Zeitschrift des ADSV, 1886-1908. Zugänglich unter: https://de.wikisource.org/wiki/Zeitschrift des_allgemeinen_deutschen_Sprachvereins [20.3.2021]. 


\section{Sekundärliteratur:}

Bahlcke, Joachim / Eberhard, Winfried / PolívKa, Miloslav (1998, Hrsg.): Böhmen und Mähren. Stuttgart.

Blume. Herbert (2013): Erfolge und Misserfolge des lexikalischen Purismus in Deutschland zur Zeit des Allgemeinen deutschen Sprachvereins und heute. In: Archiv Muttersprache 3, 2013. Zugänglich unter: https://gfds.de/erfolge-und-misserfolge-des-lexikalischen-purismus-in-deutschland-zur-zeit-des-allgemeinen-deutschen-sprachvereins-und-heute [26.02.2021].

Deutscher Kalender für die Iglauer Sprachinsel. Herausgegeben und verlegt vom Deutschen Vereine für Iglau und Umgebung 10, 1896. Iglau.

Dunger, Hermann / Lössnitzer, Ernst (1900): Deutsche Speisekarte. Verdeutschung der in der Küche und Gasthofswesen gebräuchlichen entbehrlichen Fremdwörter. 4., stark vermehrte Auflage. Berlin. (= Verdeutschungsbücher des ADSV 1)

EgGERs, Hans (1992): Deutsche Sprachgeschichte. Band 2: Das Frühneuhochdeutsche und das Neuhochdeutsche. Reinbek bei Hamburg.

ENGELHARDT, Gerhard (2001): Český a německý purismus z konce 19. století. In: Naše řeč 84, 2001, S. 235-244.

GARDT, Andreas (1998): Die Sprachgesellschaften des 17. und 18. Jahrhunderts. In: Besch, Werner / Betten, Anne / Reichmann, Oskar / Sonderegger, Stefan (Hrsg.): Sprachgeschichte. Ein Handbuch zur Geschichte der deutschen Sprache und ihrer Erforschung. 2., vollständig neu bearbeitete und erweiterte Auflage. 1. Teilband. Berlin; New York, S. 332-348.

Greule, Albrecht / Ahlvers-Liebel, Elisabeth (1986): Germanistische Sprachpflege. Darmstadt.

HARsdörfFer, Georg Philipp (1656): Der Teutsche Secretarius. Das ist: Allen Cantzleyen, Studirund Schreibstuben nützliches, fast nothwendiges und zum drittenmal vermehrtes Titular- und Formularbuch. Nürnberg.

Hauffen, Adolf (1916/1917): Der Allgemeine Deutsche Sprachverein und Deutschböhmen I,II. In: Deutsche Arbeit 16, H. 7/8 und 9, S. 296-302, 345-351.

HeIER, Anke (2012): Deutsche Fremdwortlexikologie zwischen 1800 und 2007. Zur metasprachlichen und lexikographischen Behandlung äußeren Lehnguts in Sprachkontaktwörterbüchern des Deutschen. Berlin; Boston.

Hundt, Markus (2000): Spracharbeit im 17. Jahrhundert. Studien zu Georg Philipp Harsdörffer, Justus Georg Schottelius und Christian Gueintz. Berlin; New York.

Jelínek, Milan (2007): Purismus. In: PleskalovÁ, Jana / KrČmová, Marie / VeČerka, Radoslav / Karlí, Petr (Hrsg.): Kapitoly z dějin české jazykovédné bohemistiky. Praha, S. 540-572.

KIRKNess, Alan (1998): Das Phänomen des Purismus in der Geschichte des Deutschen. In: Besch, Werner / Betten, Anne / Reichmann, Oskar / Sonderegger, Stefan (Hrsg.): Sprachgeschichte. Ein Handbuch zur Geschichte der deutschen Sprache und ihrer Erforschung. 2., vollständig neu bearbeitete und erweiterte Auflage. 1. Teilband. Berlin; New York, S. 407-416.

KIRKNESS, Alan (1984): Zur germanistischen Fremdwortbibliographie im 19./20. Jh.: Bibliographie der Fremd- und Verdeutschungswörterbücher 1800-1945. Zugänglich unter: https://ids-pub.bszbw.de/frontdoor/deliver/index/docId/6692/file/Kirkness_Zur_germanistischen_Fremdwordlexikographie_im_19_20_Jh_1984.pdf [18.03.2021].

KNÁPKOVÁ, Petra (2010): Ein Beitrag zur Kulturgeschichte Iglaus. Olomouc.

LOHMEYER, Edward (1915): Unsere Umgangssprache. Verdeutschungen der hauptsächlichsten im täglichen Leben und Verkehr gebrauchten Fremdwörter. Berlin. (= Verdeutschungsbücher des ADSV 3)

NEŠPOR, Václav (1936): Dějiny Olomouce. Brno.

Österreichisches biographisches Lexikon 1815-1950. Bd. 5. Wien 1972; Bd. 6. Wien 1974.

Polenz, Peter von (1994): Deutsche Sprachgeschichte vom Spätmittelalter bis zur Gegenwart. Bd. 2: 17. und 18. Jahrhundert. Berlin; New York.

Polenz, Peter von (1999): Deutsche Sprachgeschichte vom Spätmittelalter bis zur Gegenwart. Bd. 3: 19. und 20. Jahrhundert. Berlin; New York. 
Polenz, Peter von (2009): Geschichte der deutschen Sprache. 10. Auflage. Berlin; New York.

RIEGEL, Herman (1883): Ein Hauptstück von unserer Muttersprache. Mahnruf an alle national gesinnten Deutschen. Leipzig.

Riegel, Herman (1887): Die Sprachreinigung und ihre Gegner. Eine Erwiderung auf die Angriffe von Gildemeister, Grimm, Rümelin und Delbrück. Dresden.

Statistisches Jahrbuch der königlichen Hauptstadt Olmütz 1888. Bd. 1: Einleitungsband, bearbeitet von Wilibald Müller. Olmütz.

SzTANDARSKA, Katarzyna (2017): Verdeutschungshefte im Deutschen als Werkzeug im Kampf gegen Wörter fremder Herkunft. In: Studia niemcoznawcze 60, 2017, S. 835-846.

SzTANDARSKA, Katarzyna (2018): Verdeutschungswörterbücher mit dem Fachwortschatz in der deutschen Sprache. In: Colloquia Germanica Stetinensia 27, 2018, S. 205-220.

VoDRÁžKovÁ, Lenka (2007): Die Prager Germanistik nach 1882. Frankfurt am Main.

\section{Internetquellen:}

URL 1: Persönlichkeiten der Stadt Iglau. Zugänglich unter:

https://www.jihlava.cz/honsig-robert/d-498703 [10.03.2021]; https://www.jihlava.cz/langer-karl/ d-498743 [10.03.2021].

URL 2: Brünn und die völkische Turnbewegung. Zugänglich unter:

http://www.znaim.eu/8-Vereine/Bruenn und die voelkische Turnbewegung [13.03.2021]. 